\title{
Severe disseminated intravascular coagulation associated with massive ventricular mural thrombus following acute myocardial infarction
}

\author{
S.A. Solomon, D.W.K. Cotton, F.E. Preston and L.E. Ramsay
}

University Departments of Haematology, Pathology and Therapeutics, Royal Hallamshire Hospital, Sheffield S10 $2 J F, U K$

\begin{abstract}
Summary: We describe three patients who developed severe disseminated intravascular coagulation associated with large ventricular mural thrombi shortly after presenting with acute myocardial infarction. To our knowledge this association has not been reported before.
\end{abstract}

\section{Introduction}

Disseminated intravascular coagulation (DIC) has been reported in a wide variety of disorders, with infection, malignancy, trauma and surgery accounting for most cases. ${ }^{1}$ Modest increases in fibrin degradation products have been reported in patients with severe myocardial infarction but overt DIC is rare. ${ }^{2}$ We describe three patients who developed severe DIC associated with large ventricular thrombi shortly after presenting with acute myocardial infarction. Large intravascular thrombi arising from intra- or extracardiac sites have been reported to cause DIC.11,12 We propose that the large ventricular mural thrombi may have initiated DIC in our patients.

\section{Case reports}

\section{Case 1}

A 63 year old woman was admitted with a transmural anterior myocardial infarction. She had been fit previously apart from a partial gastrectomy for peptic ulceration. Investigation showed peak serum creatine kinase (CPK) $>2000 \mu \mathrm{mol} / 1$ (normal $<195 \mu \mathrm{mol} / \mathrm{l}$ ); peak lactate dehydrogenase (LDH) $2320 \mu \mathrm{mol} / 1$ (normal $<265 \mu \mathrm{mol} / \mathrm{l}$ ), haemoglobin $10.9 \mathrm{~g} / \mathrm{dl}$ with features of iron deficiency, platelets $423 \times 10^{9} / 1$ and blood glucose $14.0 \mathrm{mmol} / 1$. Subsequent blood glucose values were around $9.0 \mathrm{mmol} / \mathrm{l}$. During the first hospital day she had a single

Correspondence: S.A. Solomon, M.R.C.P., University Department of Therapeutics, Royal Hallamshire Hospital, Sheffield S10 2JF, UK.

Accepted: 19 May 1988 episode of ventricular fibrillation, which was corrected promptly by defibrillation, followed by episodes of supraventricular and broad-complex tachycardia. She was well on day 2 , but developed mild left ventricular failure and right lower lobe consolidation on the third day. Streptococcus pneumoniae was cultured from sputum, but blood cultures were sterile. The heart failure and pneumonia responded to diuretic and antibiotic treatment. Progress was then uncomplicated until the 12th hospital day, when she developed spontaneous bleeding from venepuncture sites and purpura. Investigations were suggestive of DIC (Table I). The blood film showed the features of microangiopathic haemolysis with numerous schistocytes, microspherocytes and polychromasia. Occasional Howel-Jolly bodies were noted.

Despite treatment with low-dose subcutaneous heparin and transfusions of whole blood and platelets she developed, over the next 5 days, slowly progressing arterial occlusion in both legs, deep vein thrombosis in both legs, severe abdominal pain, and renal failure progressing to anuria. She died 17 days after admission. The drugs administered before the onset of DIC were lignocaine, mexiletine, amiodarone, spironolactone and frusemide.

Post-mortem examination revealed a recent transmural infarct involving both ventricles, with a large lamellated mural thrombus overlying the infarct (Figure 1). All three coronary arteries were involved by moderate to severe atheroma and the bifurcation of the left coronary artery contained recent thrombus blocking both branches. The right pulmonary artery was blocked by a large embolus and there were in addition multiple peripheral thrombi and

(C) The Fellowship of Postgraduate Medicine, 1988 
Table I Results of coagulation studies in three cases

\begin{tabular}{|c|c|c|c|}
\hline \multirow[t]{2}{*}{ Investigation } & \multicolumn{3}{|c|}{ Patients } \\
\hline & Case 1 & Case 2 & Case 3 \\
\hline 1. Platelet count (normal range $150-400 \times 10^{9} / 1$ ) & 5.0 & 27.0 & 88.0 \\
\hline 2. Prothrombin time (control) (seconds) & $15.0(12.0)$ & $23.0(13.0)$ & $45.0(12.0)$ \\
\hline 3. KCCT (control) (seconds) & $38.0(40.0)$ & $58.0(42.0)$ & $63.0(41.0)$ \\
\hline 4. Thrombin time (control) (seconds) & $10.0(11.0)$ & $25.0(10.0)$ & $13.0(11.0)$ \\
\hline 5. Fibrin degradation products (normal range $0-8 \mathrm{ng} / \mathrm{ml}$ ) & 64.0 & 256.0 & 128.0 \\
\hline 6. Fibrin monomer & Positive & Positive & Positive \\
\hline 7. Fibrinogen $(\mathrm{g} / \mathrm{l})$ (normal range $1.6-3.9 \mathrm{~g} / \mathrm{l}$ ) & 1.7 & 0.3 & 1.2 \\
\hline 8. Haemoglobin $(\mathrm{g} / \mathrm{dl})$ & 8.5 & 14.8 & 12.6 \\
\hline 9. White cell count $\times 10^{9} / 1$ & 16.4 & 24.0 & 19.7 \\
\hline
\end{tabular}

infarcts. In the legs both femoral and posterior tibial veins contained extensive thrombi. The liver showed several infarcts associated with multiple hepatic vein thromboses, and several fibrin plugs were seen in the spleen (Figure 2).

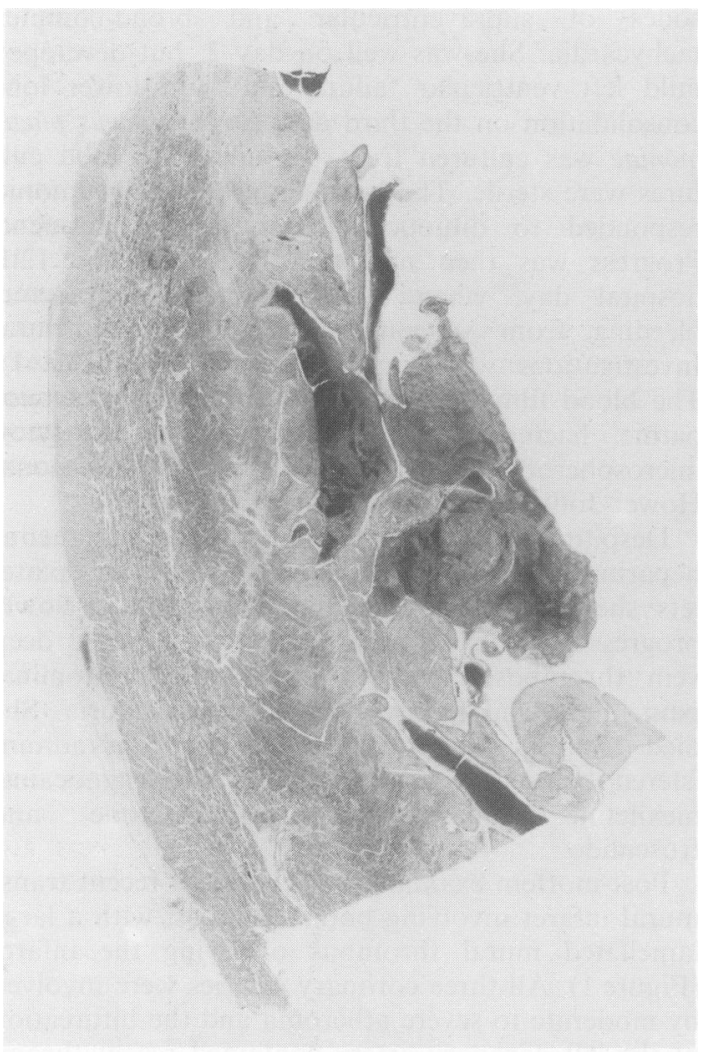

Figure 1 Low power photomicrograph of left ventricular free wall with recent infarction and laminated thrombus overlying it (Case 1). (H \& $\mathrm{E} \times 2.8)$.

\section{Case 2}

A 52 year old man was admitted with a transmural anterior myocardial infarction. He was previously fit. Serum CPK peaked at $>2000 \mu \mathrm{mol} / 1$ and $\mathrm{LDH}$

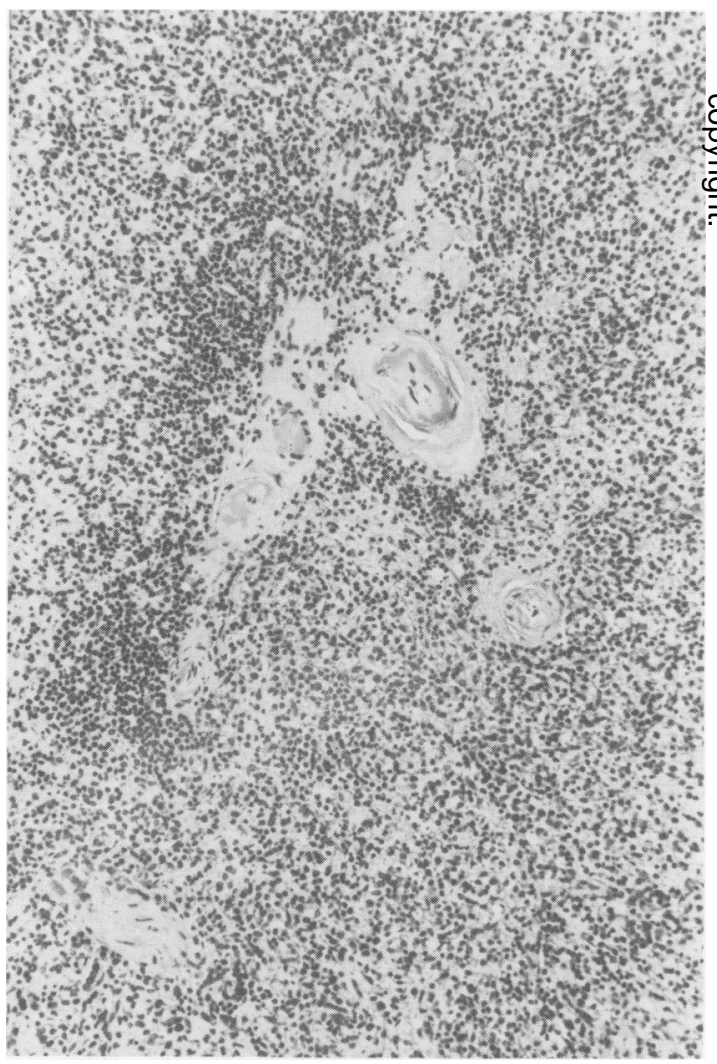

Figure 2 High power photomicrograph of the spleen with thrombin plugs in small vessels (Case 1). (H \& $\mathrm{E} \times 42.5$ ). 
at $>2280 \mu \mathrm{mol} / \mathrm{l}$. On admission haemoglobin was $14.6 \mathrm{~g} / \mathrm{dl}$; platelet count $269 \times 10^{9} / 1$, and blood glucose $12.2 \mathrm{mmol} / \mathrm{l}$.

He had a single episode of ventricular fibrillation, which responded to defibrillation, on the first hospital day. On day 2 , he had pericarditis and mild left ventricular failure which responded to diuretic treatment. Blood cultures were sterile. He was then well until day 7 when he became drowsy, generally unwell and dehydrated. Investigations revealed a hyperosmolar non-ketotic diabetic state with blood glucose $30.5 \mathrm{mmol} / 1$ and calculated serum osmolality $345 \mathrm{mosm} / \mathrm{l}$. He was treated with intravenous saline and insulin, and the blood glucose had fallen to $8.3 \mathrm{mmol} / \mathrm{l} 6$ hours later. At this time he developed spontaneous bleeding from venepuncture sites and his nose, and tests of coagulation indicated DIC (Table I). His blood film was grossly abnormal and showed the characteristic features of microangiopathic haemolysis. HowellJolly bodies were also prominent.

He was treated with cryoprecipitate and subcutaneous heparin, 5000 units 8-hourly, but developed slowly progressing arterial occlusion in both legs, renal failure progressing to anuria and worsening left ventricular failure. He died on the 12th hospital day. The drugs administered before the onset of DIC were frusemide, glibenclamide and aspirin.

Post-mortem examination showed a circumferential left ventricular infarct of 1-2 weeks duration covered by organizing thrombus. The left anterior descending coronary artery was blocked by thrombus. The liver showed evidence of chronic congestive cardiac failure and the lungs were oedematous and congested with early consolidation at the bases. Histology confirmed the myocardial infarction and revealed centrilobular necrosis in the liver. The lungs showed diffuse alveolar damage consistent with adult respiratory distress syndrome (Figure 3).

\section{Case 3}

A 61 year old man with asymptomatic mitral valve prolapse was admitted with a transmural anterior myocardial infarction. Peak serum CPK was $>2000 \mu \mathrm{mol} / 1$ and LDH $>3000 \mu \mathrm{mol} / 1$. On admission haemoglobin was $12.5 \mathrm{~g} / \mathrm{dl}$; platelet count $209 \times 10^{9} / 1$ and blood glucose $11.0 \mathrm{mmol} / 1$. He developed worsening left ventricular failure and cardiogenic shock, which did not respond to treatment, and by day 5 had renal failure (serum creatinine $469 \mathrm{mmol} / \mathrm{l}$ ) and metabolic acidosis. On the fifth day he developed purpura and spontaneous bleeding from venepuncture sites and there was unequivocal laboratory evidence of DIC (Table I). He continued to deteriorate, developed arterial occlusion in both legs and died 6 days after admis-

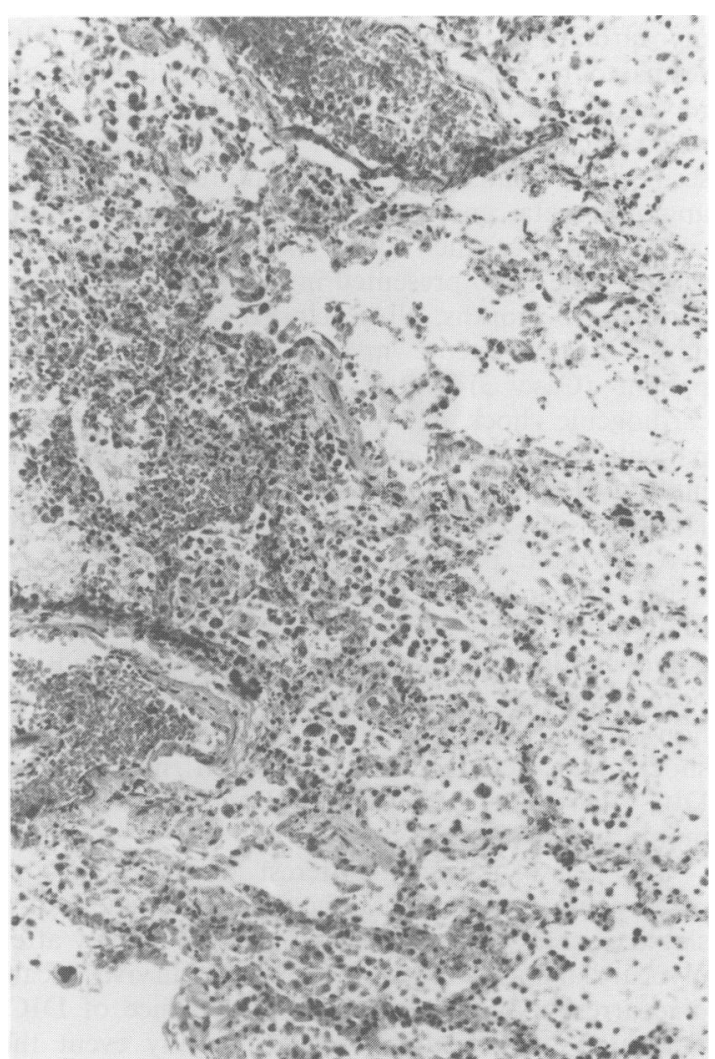

Figure 3 High power photomicrograph of lung showing debris within the alveoli and hyaline material lining their walls (Case 2). (H \& E $\times 42.5$ ).

sion. The drugs administered before the onset of DIC were frusemide, dopamine and Resonium A.

Post-mortem examination showed an established circumferential, transmural myocardial infarction of the left ventricle. There was mural thrombus overlying the infarct within each ventricle. Recent thrombus occluded the right coronary and all three coronary arteries showed moderate to severe atheroma. The mitral valve showed 'floppy mitral valve' changes with a prolapsing posterior leaflet. The lungs and liver showed evidence of acute and chronic cardiac failure respectively. Histology confirmed the macroscopic findings in the heart and revealed centrilobular necrosis in the liver. No direct evidence of small vessel occlusion was recognized.

\section{Discussion}

Modest increases of fibrinogen degradation products have been noted in some patients with severe 
myocardial infarction, but clinically overt DIC seems to be very rare. ${ }^{2}$ We have found only one report of overt DIC possibly complicating acute myocardial infarction. ${ }^{3}$ However, the patient described also had severe heart failure and multiple angiolipomata, and these were considered the principal causes of the DIC. The three patients described here, who presented in one hospital within a period of 5 months, all developed severe DIC 5-12 days after an acute myocardial infarction. One patient (Case 3) would probably have died of cardiogenic shock in any event, but the other two patients were recovering from their myocardial infarctions satisfactorily and died of overwhelming DIC. Possible causes of DIC could be identified in two of these patients. Case 2 had developed a hyperosmolar ketotic state 7 days after myocardial infarction. Severe DIC is a recognized but rare complication of severe diabetic ketoacidosis and has been reported occasionally in the hyperosmolar non-ketotic diabetic state. ${ }^{4,5}$ Case 3 had severe shock and acidosis, factors which may trigger DIC. ${ }^{6}$ However DIC has not been reported commonly in cardiogenic shock.

Case 1 is of particular interest because no recognized cause of DIC could be identified. She had a single episode of ventricular fibrillation shortly after myocardial infarction. Patients who survive a cardiac arrest may show laboratory evidence of DIC, but this is mild and transient. ${ }^{7}$ In any event the cardiac arrest occurred 11 days before the onset of DIC. She also had pneumococcal pneumonia 9 days before the onset of DIC but this had responded rapidly to antibiotic treatment and was not complicated by septicaemia.

The striking observation was that all three patients had massive ventricular mural thrombi. Mural thrombus develops in about one third of patients with anterior myocardial infarction, but the thrombi observed in these cases were exceptionally large. ${ }^{8}$ We believe that the association with severe DIC was not fortuitous. The association of DIC with massive left ventricular mural thrombus has been reported previously in one patient with heart failure. ${ }^{9}$ The authors suggested that DIC led to incorporation of fibrin into a pre-existing mural thrombus resulting in growth of the thrombus to

\section{References}

1. Colman, R.W., Robbey, J.S. \& Minnor, J.S. Disseminated intravascular coagulation (DIC): an approach. Am J Med 1972, 52: 679-689.

2. Okuno, T. \& Nelson, C. Value of determination of serum fibrin-fibrinogen degradation products in acute myocardial infarction. Am J Clin Pathol 1974, 61: 155-159. massive proportions. This interpretation is supported by evidence that DIC can cause formation of mural thrombus even when coronary thrombosis and myocardial infarction have not occurred. ${ }^{10}$ It is therefore conceivable that DIC caused growth of pre-existing mural thrombus and resulted in massive thrombus formation in our patients. However, if this was the complete explanation for the association, the development of DIC in Case 1 would remain entirely unexplained. We suggest that the massive mural thrombi actually caused, or at least contributed to, the development of DIC in our patients. In support of this is the observation that giant left atrial thrombus in mitral stenosis ${ }^{11,12}$ has been associated with DIC. One explanation is that these thrombi 'consume' coagulation factors locally, as evidenced by incorporation of fibrin and fibrinogen $^{11}$ but a more likely mechanism is that injury to red cells, platelets, or both act as a trigger to the coagulation mechanism. ${ }^{1}$ The abnormal pattern of blood flow over large intravascular thrombi may result in red cell and platelet injury, leading to activation of coagulation and platelets and widespread dispersion of activated clotting factors. ${ }^{1}$ The net result being enhanced coagulation at the surface of the thrombus and DIC. In support of this, Casen 1 , who had no recognized cause of DIC, showede fibrin plugs, thrombi, and infarcts in several organs These findings could not be explained by 'consumption' of coagulation factors. In addition the blood films of Cases 1 and 2 showed the presence of large numbers of schistocytes which provide good supportive evidence of DIC. It is well established that these are red cells that have sustained mechanical damage on thin fibrin strands. ${ }^{13}$

We propose that formation of large ventricular mural thrombi may have initiated the DIC in our patients. DIC may then have caused further enlargement of the mural thrombi with consequent worsening of DIC.

\section{Acknowledgements}

We thank Dr C.D. Holdsworth and Dr J.J. Daly for allowing us to report cases under their care, $\mathrm{Dr} \mathrm{N}$. Rooney and Dr S. Dundas for the histopathology reports and Mrs E. Grassam for typing the manuscript.

3. Rustin, G.J.S. Diffuse intravascular coagulation in association with myocardial infarction and multiple angiolipomata. Postgrad Med J 1977, 53: 228-229.

4. Kwaan, H.C., Colwell, J.A. \& Suwanwela, N. Disseminated intravascular coagulation in diabetes mellitus, with reference to the role of increased platelet aggregation. Diabetes 1972, 21: 108-113. 
5. Timperley, W.R., Preston, F.E. \& Ward, J.D. Cerebral intravascular coagulation in diabetic ketoacidosis. Lancet 1974; i: 952-956.

6. Hardaway, R.M., James, P.M., Anderson, R.W. et al. Intensive study and treatment of shock in man. JAMA 1967, 99: 779-790.

7. Mehta, B., Briggs, D.K., Sommers, S.C. \& Karpatkin, M. Disseminated intravascular coagulation following cardiac arrest: a study of 15 patients. Am J Med Sci 1972, 264: 353-363.

8. Asinger, R.W., Mikell, F.L., Elsperger, J. \& Hodges, M. Incidence of left ventricular thrombosis after acute transmural myocardial infarction. $N$ Engl J Med 1981, 305: 297-302.

9. Heckman, T.A. \& Tosove, M.H. Massive left ventricular mural thrombosis with consumption coagulopathy in congestive heart failure. West $\mathrm{J}$ Med 1980, 133: 442-444.
10. Sigiura, M., Hiroaka, K., Ohkawa, S., Ueda, K., Malsuda, J. \& Murakami M. A clinicopathological study on cardiac lesions in 64 cases of disseminated intravascular coagulation. Jpn Heart $J$ 1977, 18: 57-69.

11. Ikematsu, S., Itoh, O., Samori, T. et al. A case of consumption coagulopathy due to atrial giant thrombus. Rinsho Ketsueki 1975, 16: 530-536.

12. McIlraith, D.M., Mount, M.J. \& Brien, F.W. Chronic consumptive coagulopathy due to intracardiac thrombus. Am J Med 1987, 82: 135-136.

13. Bull, B.S., Rubenberg, M.L., Dacie, J.V. \& Brain, M.C. Microangiopathic haemolytic anaemia; mechanisms of red-cell fragmentation: in vitro studies. Br J Haematol 1968, 14: 643-652. 